Research Article

\title{
Stability of a Jensen Type Logarithmic Functional Equation on Restricted Domains and Its Asymptotic Behaviors
}

\author{
Jae-Young Chung \\ Department of Mathematics, Kunsan National University, Kunsan 573-701, Republic of Korea \\ Correspondence should be addressed to Jae-Young Chung, jychung@kunsan.ac.kr \\ Received 28 June 2010; Revised 30 October 2010; Accepted 25 December 2010 \\ Academic Editor: Roderick Melnik
}

Copyright (c) 2010 Jae-Young Chung. This is an open access article distributed under the Creative Commons Attribution License, which permits unrestricted use, distribution, and reproduction in any medium, provided the original work is properly cited.

Let $\mathbb{R}_{+}$be the set of positive real numbers, $B$ a Banach space, $f: \mathbb{R}_{+} \rightarrow B$, and $\epsilon>0, p, q, P, Q \in$ $\mathbb{R}$ with $p q P Q \neq 0$. We prove the Hyers-Ulam stability of the Jensen type logarithmic functional inequality $\left\|f\left(x^{p} y^{q}\right)-P f(x)-Q f(y)\right\| \leq \epsilon$ in restricted domains of the form $\{(x, y): x>0, y>$ $\left.0, x^{k} y^{s} \geq d\right\}$ for fixed $k, s \in \mathbb{R}$ with $k \neq 0$ or $s \neq 0$ and $d>0$. As consequences of the results we obtain asymptotic behaviors of the inequality as $x^{k} y^{s} \rightarrow \infty$.

\section{Introduction}

The stability problems of functional equations have been originated by Ulam in 1940 (see

[1]). One of the first assertions to be obtained is the following result, essentially due to Hyers [2], that gives an answer for the question of Ulam.

Theorem 1.1. Suppose that $\langle S,+\rangle$ is an additive semigroup, $B$ is a Banach space, $\epsilon \geq 0$, and $f: S \rightarrow$ $B$ satisfies the inequality

$$
\|f(x+y)-f(x)-f(y)\| \leq \epsilon
$$

for all $x, y \in S$. Then there exists a unique function $A: S \rightarrow B$ satisfying

$$
A(x+y)=A(x)+A(y)
$$


for which

$$
\|f(x)-A(x)\| \leq \epsilon
$$

for all $x \in S$.

In 1950-1951 this result was generalized by the authors Aoki [3] and Bourgin [4, 5]. Unfortunately, no results appeared until 1978 when Th. M. Rassias generalized the Hyers' result to a new approximately linear mappings [6]. Following the Rassias' result, a great number of the papers on the subject have been published concerning numerous functional equations in various directions [6-16]. For more precise descriptions of the Hyers-Ulam stability and related results, we refer the reader to the paper of Moszner [17]. Among the results, the stability problem in a restricted domain was investigated by Skof, who proved the stability problem of the inequality (1.1) in a restricted domain [16]. Developing this result, Jung considered the stability problems in restricted domains for the Jensen functional equation [11] and Jensen type functional equations [14]. The results can be summarized as follows: let $X$ and $Y$ be a real normed space and a real Banach space, respectively. For fixed $d>0$, if $f: X \rightarrow Y$ satisfies the functional inequalities (such as that of Cauchy, Jensen and Jensen type, etc.) for all $x, y \in X$ with $\|x\|+\|y\| \geq d$, the inequalities hold for all $x, y \in X$. We also refer the reader to [18-26] for some interesting results on functional equations and their Hyers-Ulam stabilities in restricted conditions.

Throughout this paper, we denote by $\mathbb{R}_{+}$the set of positive real numbers, $B$ a Banach space, $f: \mathbb{R}_{+} \rightarrow B$, and $p, q, P, Q \in \mathbb{R}$ with $p q P Q \neq 0$. We prove the Hyers-Ulam stability of the Jensen type logarithmic functional inequality

$$
\left\|f\left(x^{p} y^{q}\right)-P f(x)-Q f(y)\right\| \leq \epsilon
$$

in the restricted domains of the form $U_{k, s}=\left\{(x, y): x>0, y>0, x^{k} y^{s} \geq d\right\}$ for fixed $k, s \in \mathbb{R}$ with $k \neq 0$ or $s \neq 0$, and $d>0$. As a result, we prove that if the inequality (1.4) holds for all $(x, y) \in U_{k, s}$, there exists a unique function $L: \mathbb{R}_{+} \rightarrow B$ satisfying

$$
L(x y)-L(x)-L(y)=0, \quad x, y>0
$$

for which

$$
\|f(x)-L(x)-f(1)\| \leq 4 \epsilon
$$

for all $x>0$ if $k / p \neq s / q$,

$$
\|f(x)-L(x)-f(1)\| \leq \frac{4 \epsilon}{|P|}
$$

for all $x>0$ if $s \neq 0$, and

$$
\|f(x)-L(x)-f(1)\| \leq \frac{4 \epsilon}{|Q|}
$$


for all $x>0$ if $k \neq 0$. As a consequence of the result we obtain the stability of the inequality

$$
\|f(p x+q y)-P f(x)-Q f(y)\| \leq \epsilon
$$

in the restricted domains of the form $\left\{(x, y) \in \mathbb{R}^{2}: k x+s y \geq d\right\}$ for fixed $k, s \in \mathbb{R}$ with $k \neq 0$ or $s \neq 0$, and $d \in \mathbb{R}$. Also we obtain asymptotic behaviors of the inequalities (1.4) and (1.9) as $x^{k} y^{s} \rightarrow \infty$ and $k x+s y \rightarrow \infty$, respectively.

\section{Hyers-Ulam Stability in Restricted Domains}

We call the functions satisfying (1.5) logarithmic functions. As a direct consequence of Theorem 1.1, we obtain the stability of the logarithmic functional equation, viewing $\left\langle\mathbb{R}_{+}, x\right\rangle$ as a multiplicative group (see also the result of Forti [9]).

Theorem A. Suppose that $f: \mathbb{R}_{+} \rightarrow B, \epsilon \geq 0$, and

$$
\|f(x y)-f(x)-f(y)\| \leq \epsilon
$$

for all $x, y>0$. Then there exists a unique logarithmic function $L: \mathbb{R}_{+} \rightarrow B$ satisfying

$$
\|f(x)-L(x)\| \leq \epsilon
$$

for all $x>0$.

We first consider the usual logarithmic functional inequality (2.1) in the restricted domains $U_{k, s}$.

Theorem 2.1. Let $\epsilon, d>0, k, s \in \mathbb{R}$ with $k \neq 0$ or $s \neq 0$. Suppose that $f: \mathbb{R}_{+} \rightarrow B$ satisfies

$$
\|f(x y)-f(x)-f(y)\| \leq \epsilon
$$

for all $x, y>0$, with $x^{k} y^{s} \geq d$. Then there exists a unique logarithmic function $L: \mathbb{R}_{+} \rightarrow B$ such that

$$
\|f(x)-L(x)\| \leq 3 \epsilon
$$

for all $x \in \mathbb{R}_{+}$. 
Proof. From the symmetry of the inequality we may assume that $s \neq 0$. For given $x, y \in \mathbb{R}_{+}$, choose a $z>0$ such that $x^{k} y^{k} z^{s} \geq d, x^{k} y^{s} z^{s} \geq d$, and $y^{k} z^{s} \geq d$. Then we have

$$
\begin{aligned}
\|f(x y)-f(x)-f(y)\| \leq & \|-f(x y z)+f(x y)+f(z)\| \\
& +\|f(x y z)-f(x)-f(y z)\| \\
& +\|f(y z)-f(y)-f(z)\|
\end{aligned}
$$

$\leq 3 \epsilon$.

This completes the proof.

Now we consider the Hyers-Ulam stability of the Jensen type logarithmic functional inequality (1.4) in the restricted domains $U_{k, s}$.

Theorem 2.2. Let $\epsilon, d>0, k, s \in \mathbb{R}, k / p \neq s / q$. Suppose that $f: \mathbb{R}_{+} \rightarrow B$ satisfies

$$
\left\|f\left(x^{p} y^{q}\right)-P f(x)-Q f(y)\right\| \leq \epsilon
$$

for all $x, y>0$, with $x^{k} y^{s} \geq d$. Then there exists a unique logarithmic function $L: \mathbb{R}_{+} \rightarrow B$ such that

$$
\|f(x)-L(x)-f(1)\| \leq 4 \epsilon
$$

for all $x \in R_{+}$.

Proof. Replacing $x$ by $x^{1 / p}, y$ by $y^{1 / q}$ in (2.6) we have

$$
\left\|f(x y)-P f\left(x^{1 / p}\right)-Q f\left(y^{1 / q}\right)\right\| \leq \epsilon
$$

for all $x, y>0$, with $x^{k / p} y^{s / q} \geq d$.

For given $x, y \in \mathbb{R}_{+}$, choose a $z>0$ such that $x^{k / p} y^{s / q} z^{s / q-k / p} \geq d, x^{k / p} z^{s / q-k / p} \geq d$, $y^{s / q} z^{s / q-k / p} \geq d$, and $z^{s / q-k / p} \geq d$. Replacing $x$ by $x z^{-1}, y$ by $y z ; x$ by $x z^{-1}, y$ by $z ; x$ by $z^{-1}, y$ by $y z ; x$ by $z^{-1}, y$ by $z$ in (2.8) we have

$$
\begin{aligned}
\|f(x y)-f(x)-f(y)+f(1)\| \leq & \left\|f(x y)-P f\left(x^{1 / p} z^{-1 / p}\right)-Q f\left((y z)^{1 / q}\right)\right\| \\
& +\left\|-f(x)+P f\left(x^{1 / p} z^{-1 / p}\right)+Q f\left(z^{1 / q}\right)\right\| \\
& +\left\|-f(y)+P f\left(z^{-1 / p}\right)+Q f\left((y z)^{1 / q}\right)\right\| \\
& +\left\|f(1)-P f\left(z^{-1 / p}\right)-Q f\left(z^{1 / q}\right)\right\| \\
\leq & 4 e .
\end{aligned}
$$


Now by Theorem A, there exists a unique logarithmic function $L: \mathbb{R}_{+} \rightarrow B$ such that

$$
\|f(x)-L(x)-f(1)\| \leq 4 \epsilon
$$

for all $x \in \mathbb{R}_{+}$. This completes the proof.

As a matter of fact, we obtain that $L=0$ in Theorem 2.2 provided that $p \neq P$ and $p$ or $P$ is a rational number, or $q \neq Q$ and $q$ or $Q$ is a rational number.

Theorem 2.3. Let $\epsilon, d>0, k, s \in \mathbb{R}, k / p \neq s / q$. Suppose that $p \neq P$ and $p$ or $P$ is a rational number, or $q \neq Q$ and $q$ or $Q$ is a rational number, and $f: \mathbb{R}_{+} \rightarrow B$ satisfies

$$
\left\|f\left(x^{p} y^{q}\right)-P f(x)-Q f(y)\right\| \leq \epsilon
$$

for all $x, y>0$, with $x^{k} y^{s} \geq d$. Then one has

$$
\|f(x)-f(1)\| \leq 4 \epsilon
$$

for all $x \in \mathbb{R}_{+}$.

Proof. We prove (2.12) only for the case that $p \neq P$ and $p$ or $P$ is a rational number since the other case is similarly proved. From (2.7) and (2.11), using the triangle inequality we have

$$
\left\|L\left(x^{p} y^{q}\right)-P L(x)-Q L(y)\right\| \leq M
$$

for all $x, y>0$, with $x^{k} y^{s} \geq d$, where $M=\epsilon(5+4|P|+4|Q|)+|f(1)(1-P-Q)|$. If $k \neq 0$, putting $y=1$ in (2.13) we have

$$
\left\|L\left(x^{p}\right)-P L(x)\right\| \leq M
$$

for all $x>0$, with $x^{k} \geq d$. It is easy to see that $L\left(x^{r}\right)=r L(x)$ for all $x>0$ and all rational numbers $r$. Thus if $p$ is a rational number, it follows from (2.14) that

$$
\|L(x)\| \leq \frac{M}{|p-P|}
$$

for all $x>0$, with $x^{k} \geq d$. If there exists $x_{0}>0$ such that $L\left(x_{0}\right) \neq 0$, we can choose a rational number $r$ such that $x_{0}^{r k} \geq d$ and $\left\|r L\left(x_{0}\right)\right\|>M /|p-P|$ (it is realized when $r$ is large if $x_{0}^{k}>1$, and when $-r$ is large if $x_{0}^{k}<1$ ). Now we have

$$
\frac{M}{|p-P|}<\left\|r L\left(x_{0}\right)\right\|=\left\|L\left(x_{0}^{r}\right)\right\| \leq \frac{M}{|p-P|} .
$$


Thus it follows that $L=0$. If $P$ is a rational number, it follows from (2.14) that

$$
\left\|L\left(x^{p-P}\right)\right\| \leq M
$$

for all $x>0$, with $x^{k} \geq d$, which implies

$$
\|L(x)\| \leq M
$$

for all $x>0$, with $x^{k /(p-P)} \geq d$. Similarly, using (2.18) we can show that $L=0$. If $k=0$, choosing $y_{0}>0$ such that $y_{0}^{s} \geq d$, putting $y=y_{0}$ in (2.13) and using the triangle inequality we have

$$
\left\|L\left(x^{p}\right)-P L(x)\right\| \leq M+\left|L\left(y_{0}^{q}\right)-Q L\left(y_{0}\right)\right|
$$

for all $x>0$. Similarly, using (2.19) we can show that $L=0$. Thus the inequality (2.12) follows from (2.7). This completes the proof.

Theorem 2.4. Let $\epsilon, d>0, k, s \in \mathbb{R}$ with $k \neq 0$ or $s \neq 0$. Suppose that $f: \mathbb{R}_{+} \rightarrow B$ satisfies

$$
\left\|f\left(x^{p} y^{q}\right)-P f(x)-Q f(y)\right\| \leq \epsilon
$$

for all $x, y>0$, with $x^{k} y^{s} \geq d$. Then there exists a unique logarithmic function $L: \mathbb{R}_{+} \rightarrow B$ such that

$$
\|f(x)-L(x)-f(1)\| \leq \frac{4 \epsilon}{|P|}
$$

for all $x \in \mathbb{R}_{+}$if $s \neq 0$, and

$$
\|f(x)-L(x)-f(1)\| \leq \frac{4 \epsilon}{|Q|}
$$

for all $x \in \mathbb{R}_{+}$if $k \neq 0$. 
Proof. Assume that $s \neq 0$. For given $x, y \in \mathbb{R}_{+}$, choose a $z>0$ such that $x^{k} y^{k} z^{s} \geq d, x^{k} y^{p s / q} z^{s} \geq$ $d, y^{k} z^{s} \geq d$ and $y^{p s / q} z^{s} \geq d$. Replacing $x$ by $x y, y$ by $z ; x$ by $x, y$ by $y^{p / q} z ; x$ by $y, y$ by $z ; x$ by $1, y$ by $y^{p / q} z$ in (2.20) we have

$$
\begin{aligned}
\|P f(x y)-P f(x)-P f(y)+P f(1)\| \leq & \left\|-f\left((x y)^{p} z^{q}\right)+P f(x y)+Q f(z)\right\| \\
& +\left\|f\left((x y)^{p} z^{q}\right)-P f(x)-Q f\left(y^{p / q} z\right)\right\| \\
& +\left\|f\left(y^{p} z^{q}\right)-P f(y)-Q f(z)\right\| \\
& +\left\|-f\left(y^{p} z^{q}\right)+P f(1)+Q f\left(y^{p / q} z\right)\right\|
\end{aligned}
$$

$\leq 4 \epsilon$.

Dividing (2.23) by $|P|$ and using Theorem A, we obtain that there exists a unique logarithmic function $L: \mathbb{R}_{+} \rightarrow B$ such that

$$
\|f(x)-L(x)-f(1)\| \leq \frac{4 \epsilon}{|P|}
$$

for all $x \in \mathbb{R}_{+}$. Assume that $k \neq 0$. For given $x, y \in \mathbb{R}_{+}$, choose a $z>0$ such that $x^{s} y^{s} z^{k} \geq$ $d, x^{q k / p} y^{s} z^{k} \geq d, x^{s} z^{k} \geq d$ and $x^{q k / p} z^{k} \geq d$. Replacing $y$ by $x y, x$ by $z ; y$ by $y, x$ by $x^{q / p} z ; y$ by $x, x$ by $z ; y$ by $1, x$ by $x^{q / p} z$ in (2.20) we have

$$
\begin{aligned}
\|Q f(x y)-Q f(x)-Q f(y)+Q f(1)\| \leq & \left\|-f\left((x y)^{q} z^{p}\right)+P f(z)+Q f(x y)\right\| \\
& +\left\|f\left((x y)^{q} z^{p}\right)-P f\left(x^{q / p} z\right)-Q f(y)\right\| \\
& +\left\|f\left(x^{q} z^{p}\right)-P f(z)-Q f(x)\right\| \\
& +\left\|-f\left(x^{q} z^{p}\right)+P f\left(x^{q / p} z\right)+Q f(1)\right\| \\
\leq & 4 \epsilon .
\end{aligned}
$$

Dividing (2.25) by $|Q|$ and using Theorem A, we obtain that there exists a unique logarithmic function $L: \mathbb{R}_{+} \rightarrow B$ such that

$$
\|f(x)-L(x)-f(1)\| \leq \frac{4 \epsilon}{|Q|}
$$

for all $x \in \mathbb{R}_{+}$. This completes the proof.

From Theorem 2.4, using the same approach as in the proof of Theorem 2.3 we have the following. 
Theorem 2.5. Let $\epsilon, d>0, k, s \in \mathbb{R}$ with $k \neq 0$ or $s \neq 0$. Suppose that $p \neq P$ and $p$ or $P$ is a rational number, or $q \neq Q$ and $q$ or $Q$ is a rational number, and $f: \mathbb{R}_{+} \rightarrow B$ satisfies

$$
\left\|f\left(x^{p} y^{q}\right)-P f(x)-Q f(y)\right\| \leq \epsilon
$$

for all $x, y>0$, with $x^{k} y^{s} \geq d$. Then one has

$$
\|f(x)-f(1)\| \leq \frac{4 \epsilon}{|P|}
$$

for all $x \in \mathbb{R}_{+}$if $s \neq 0$, and

$$
\|f(x)-f(1)\| \leq \frac{4 \epsilon}{|Q|}
$$

for all $x \in \mathbb{R}_{+}$if $k \neq 0$.

We call $A: \mathbb{R} \rightarrow B$ an additive function provided that

$$
A(x+y)=A(x)+A(y)
$$

for all $x, y \in \mathbb{R}$. Using Theorem 2.2 we have the following.

Corollary 2.6 (see [22]). Let $\epsilon>0, d, k, s \in \mathbb{R}$ with $k / p \neq s / q$. Suppose that $g: \mathbb{R} \rightarrow B$ satisfies

$$
\|g(p x+q y)-P g(x)-Q g(y)\| \leq \epsilon
$$

for all $x, y \in \mathbb{R}$, with $k x+s y \geq d$. Then there exists a unique additive function $A: \mathbb{R} \rightarrow B$ such that

$$
\|g(x)-A(x)-g(0)\| \leq 4 \epsilon
$$

for all $x \in \mathbb{R}$.

Proof. Replacing $x$ by $\ln u, y$ by $\ln v$ in (2.31) and setting $f(x)=g(\ln x)$ we have

$$
\left\|f\left(u^{p} v^{q}\right)-P f(u)-Q f(v)\right\| \leq \epsilon
$$

for all $u, v \in \mathbb{R}$, with $u^{k} v^{s} \geq e^{d}$. Using Theorem 2.2, we have

$$
\|f(x)-L(x)-f(1)\| \leq 4 \epsilon
$$

for all $x \in \mathbb{R}_{+}$, which implies

$$
\left\|g(x)-L\left(e^{x}\right)-g(0)\right\| \leq 4 \epsilon
$$

for all $x \in \mathbb{R}$. Letting $A(x)=L\left(e^{x}\right)$ we get the result. 
Using Theorem 2.3, we have the following.

Corollary 2.7. Let $\epsilon>0, d, k, s \in \mathbb{R}$ with $k / p \neq s / q$. Suppose that $p \neq P$ and $p$ or $P$ is a rational number, or $q \neq Q$ and $q$ or $Q$ is a rational number, and $g: \mathbb{R} \rightarrow B$ satisfies

$$
\|g(p x+q y)-P g(x)-Q g(y)\| \leq \epsilon
$$

for all $x, y \in \mathbb{R}$, with $k x+s y \geq d$. Then one has

$$
\|g(x)-g(0)\| \leq 4 \epsilon
$$

for all $x \in \mathbb{R}$.

Using Theorem 2.4, we have the following.

Corollary 2.8. Let $\epsilon>0, d, k, s \in \mathbb{R}$ with $k \neq 0$ or $s \neq 0$. Suppose that $g: \mathbb{R} \rightarrow B$ satisfies

$$
\|g(p x+q y)-P g(x)-Q g(y)\| \leq \epsilon
$$

for all $x, y \in \mathbb{R}$, with $k x+s y \geq d$. Then there exists a unique additive function $A: \mathbb{R} \rightarrow B$ such that

$$
\|g(x)-A(x)-g(0)\| \leq \frac{4 \epsilon}{|P|}
$$

for all $x \in \mathbb{R}$ if $s \neq 0$, and

$$
\|g(x)-A(x)-g(0)\| \leq \frac{4 \epsilon}{|Q|}
$$

for all $x \in \mathbb{R}$ if $k \neq 0$.

Using Theorem 2.5, we have the following.

Corollary 2.9. Let $\epsilon>0, d, k, s \in \mathbb{R}$ with $k \neq 0$ or $s \neq 0$. Suppose that $p \neq P$ and $p$ or $P$ is a rational number, or $q \neq Q$ and $q$ or $Q$ is a rational number, and $g: \mathbb{R} \rightarrow B$ satisfies

$$
\|g(p x+q y)-P g(x)-Q g(y)\| \leq \epsilon
$$

for all $x, y \in \mathbb{R}$, with $k x+s y \geq d$. Then one has

$$
\|g(x)-g(0)\| \leq \frac{4 \epsilon}{|P|}
$$

for all $x \in \mathbb{R}$ if $s \neq 0$, and

$$
\|g(x)-g(0)\| \leq \frac{4 \epsilon}{|Q|}
$$

for all $x \in \mathbb{R}$ if $k \neq 0$. 


\section{Asymptotic Behavior of the Inequality}

In this section, we consider asymptotic behaviors of the inequalities (1.4) and (2.1).

Theorem 3.1. Let $k, s \in \mathbb{R}$ satisfy one of the conditions; $k \neq 0, s \neq 0$. Suppose that $f: \mathbb{R}_{+} \rightarrow B$ satisfies the asymptotic condition

$$
\|f(x y)-f(x)-f(y)\| \longrightarrow 0
$$

as $x^{k} y^{s} \rightarrow \infty$. Then $f$ is a logarithmic function.

Proof. By the condition (3.1), for each $n \in \mathbb{N}$, there exists $d_{n}>0$ such that

$$
\|f(x y)-f(x)-f(y)\| \leq \frac{1}{n}
$$

for all $x, y>0$, with $x^{k} y^{s} \geq d_{n}$. By Theorem 2.1, there exists a unique logarithmic function $L_{n}: \mathbb{R}_{+} \rightarrow B$ such that

$$
\left\|f(x)-L_{n}(x)\right\| \leq \frac{3}{n}
$$

for all $x \in \mathbb{R}_{+}$. From (3.4) we have

$$
\left\|L_{n}(x)-L_{m}(x)\right\| \leq \frac{3}{n}+\frac{3}{m} \leq 6
$$

for all $x \in \mathbb{R}_{+}$and all positive integers $n, m$. Now, the inequality (3.4) implies $L_{n}=L_{m}$. Indeed, for all $x>0$ and rational numbers $r>0$ we have

$$
\left\|L_{n}(x)-L_{m}(x)\right\|=\frac{1}{r}\left\|L_{n}\left(x^{r}\right)-L_{m}\left(x^{r}\right)\right\| \leq \frac{6}{r} .
$$

Letting $r \rightarrow \infty$ in (3.5), we have $L_{n}=L_{m}$. Thus, letting $n \rightarrow \infty$ in (3.3), we get the result.

Theorem 3.2. Let $k, s \in \mathbb{R}$ satisfy one of the conditions; $k \neq 0, s \neq 0, k / p \neq s / q$. Suppose that $f$ : $\mathbb{R}_{+} \rightarrow B$ satisfies the asymptotic condition

$$
\left\|f\left(x^{p} y^{q}\right)-P f(x)-Q f(y)\right\| \longrightarrow 0
$$

as $x^{k} y^{s} \rightarrow \infty$. Then there exists a unique logarithmic function $L: \mathbb{R}_{+} \rightarrow B$ such that

$$
f(x)=L(x)+f(1)
$$

for all $x \in \mathbb{R}_{+}$. 
Proof. By the condition (3.6), for each $n \in \mathbb{N}$, there exists $d_{n}>0$ such that

$$
\left\|f\left(x^{p} y^{q}\right)-P f(x)-Q f(y)\right\| \leq \frac{1}{n}
$$

for all $x, y>0$, with $x^{k} y^{s} \geq d_{n}$. By Theorems 2.2 and 2.4, there exists a unique logarithmic function $L_{n}: \mathbb{R}_{+} \rightarrow B$ such that

$$
\left\|f(x)-L_{n}(x)-f(1)\right\| \leq \frac{4}{n}
$$

if $k / p \neq s / q$,

$$
\left\|f(x)-L_{n}(x)-f(1)\right\| \leq \frac{4}{n|P|}
$$

if $s \neq 0$, and

$$
\left\|f(x)-L_{n}(x)-f(1)\right\| \leq \frac{4}{n|Q|}
$$

if $k \neq 0$. For all cases (3.9), (3.10), and (3.11), there exists $M>0$ such that

$$
\left\|L_{n}(x)-L_{m}(x)\right\| \leq M
$$

for all $x \in \mathbb{R}_{+}$and all positive integers $n, m$. Now as in the proof of Theorem 3.1, it follows from (3.12) that $L_{n}=L_{m}$ for all $n, m \in \mathbb{N}$. Letting $n \rightarrow \infty$ in (3.9), (3.10), and (3.11) we get the result.

Similarly using Theorems 2.3 and 2.5 , we have the following.

Theorem 3.3. Let $k, s \in \mathbb{R}$ satisfy one of the conditions; $k \neq 0, s \neq 0, k / p \neq s / q$. Suppose that $p \neq P$ and $p$ or $P$ is a rational number, or $q \neq Q$ and $q$ or $Q$ is a rational number, and $f: \mathbb{R}_{+} \rightarrow B$ satisfies the asymptotic condition

$$
\left\|f\left(x^{p} y^{q}\right)-P f(x)-Q f(y)\right\| \longrightarrow 0
$$

as $x^{k} y^{s} \rightarrow \infty$. Then $f$ is a constant function. 
Using Corollaries 2.6 and 2.8 we have the following.

Corollary 3.4. Let $\epsilon>0, k, s \in \mathbb{R}$ satisfy one of the conditions $k \neq 0, s \neq 0$, or $k / p \neq s / q$. Suppose that $g: \mathbb{R} \rightarrow B$ satisfies

$$
\|g(p x+q y)-P g(x)-Q g(y)\| \longrightarrow 0
$$

as $k x+$ sy $\rightarrow \infty$. Then there exists a unique additive function $A: \mathbb{R} \rightarrow B$ such that

$$
g(x)=A(x)+g(0)
$$

for all $x \in \mathbb{R}$.

Using Corollaries 2.7 and 2.9 we have the following.

Corollary 3.5. Let $\epsilon>0, k, s \in \mathbb{R}$ satisfy one of the conditions $k \neq 0, s \neq 0$, or $k / p \neq s / q$. Suppose that $p \neq P$ and $p$ or $P$ is a rational number, or $q \neq Q$ and $q$ or $Q$ is a rational number, and $g: \mathbb{R} \rightarrow B$ satisfies

$$
\|g(p x+q y)-P g(x)-Q g(y)\| \longrightarrow 0
$$

as $k x+s y \rightarrow \infty$. Then $g$ is a constant function.

\section{Acknowledgments}

The author expresses his sincere gratitude to a referee of the paper for many useful comments and introducing the interesting related recent results including the papers [17-26]. This work was supported by Basic Science Research Program through the National Research Foundation of Korea (NRF) funded by the Ministry of Education, Science and Technology (MEST) (no. 2010-0016963).

\section{References}

[1] S. M. Ulam, A Collection of Mathematical Problems, Interscience, New York, NY, USA, 1960.

[2] D. H. Hyers, "On the stability of the linear functional equation," Proceedings of the National Academy of Sciences of the United States of America, vol. 27, pp. 222-224, 1941.

[3] T. Aoki, "On the stability of the linear transformation in Banach spaces," Journal of the Mathematical Society of Japan, vol. 2, pp. 64-66, 1950.

[4] D. G. Bourgin, "Multiplicative transformations," Proceedings of the National Academy of Sciences of the United States of America, vol. 36, pp. 564-570, 1950.

[5] D. G. Bourgin, "Classes of transformations and bordering transformations," Bulletin of the American Mathematical Society, vol. 57, pp. 223-237, 1951.

[6] T. M. Rassias, "On the stability of the linear mapping in Banach spaces," Proceedings of the American Mathematical Society, vol. 72, no. 2, pp. 297-300, 1978.

[7] J. Chung, "A distributional version of functional equations and their stabilities," Nonlinear Analysis: Theory, Methods \& Applications, vol. 62, no. 6, pp. 1037-1051, 2005.

[8] J. Chung, "Stability of approximately quadratic Schwartz distributions," Nonlinear Analysis: Theory, Methods \& Applications, vol. 67, no. 1, pp. 175-186, 2007. 
[9] G. L. Forti, "The stability of homomorphisms and amenability, with applications to functional equations," Abhandlungen aus dem Mathematischen Seminar der Universität Hamburg, vol. 57, pp. 215226, 1987.

[10] D. H. Hyers, G. Isac, and T. M. Rassias, Stability of Functional Equations in Several Variables, vol. 34 of Progress in Nonlinear Differential Equations and their Applications, Birkhäuser, Boston, Mass, USA, 1998.

[11] S.-M. Jung, "Hyers-Ulam-Rassias stability of Jensen's equation and its application," Proceedings of the American Mathematical Society, vol. 126, no. 11, pp. 3137-3143, 1998.

[12] K.-W. Jun and H.-M. Kim, "Stability problem for Jensen-type functional equations of cubic mappings," Acta Mathematica Sinica, vol. 22, no. 6, pp. 1781-1788, 2006.

[13] G. H. Kim and Y. W. Lee, "Boundedness of approximate trigonometric functional equations," Applied Mathematics Letters, vol. 31, no. 4, pp. 439-443, 2009.

[14] J. M. Rassias, “On the Ulam stability of mixed type mappings on restricted domains," Journal of Mathematical Analysis and Applications, vol. 276, no. 2, pp. 747-762, 2002.

[15] J. M. Rassias and M. J. Rassias, "On the Ulam stability of Jensen and Jensen type mappings on restricted domains," Journal of Mathematical Analysis and Applications, vol. 281, no. 2, pp. 516-524, 2003.

[16] F. Skof, "Sull'approssimazione delle applicazioni localmente $\delta$-additive," Atti della Reale Accademia delle Scienze di Torino. Classe di Scienze Fisiche, Matematiche e Naturali, vol. 117, pp. 377-389, 1983.

[17] Z. Moszner, "On the stability of functional equations," Aequationes Mathematicae, vol. 77, no. 1-2, pp. 33-88, 2009.

[18] B. Batko, "Stability of an alternative functional equation," Journal of Mathematical Analysis and Applications, vol. 339, no. 1, pp. 303-311, 2008.

[19] B. Batko, "On approximation of approximate solutions of Dhombres' equation," Journal of Mathematical Analysis and Applications, vol. 340, no. 1, pp. 424-432, 2008.

[20] J. Brzdęk, "On the quotient stability of a family of functional equations," Nonlinear Analysis: Theory, Methods \& Applications, vol. 71, no. 10, pp. 4396-4404, 2009.

[21] J. Brzdęk, "On a method of proving the Hyers-Ulam stability of functional equations on restricted domains," The Australian Journal of Mathematical Analysis and Applications, vol. 6, no. 1, pp. 1-10, 2009.

[22] J. Brzdęk, "On stability of a family of functional equations," Acta Mathematica Hungarica, vol. 128, no. 1-2, pp. 139-149, 2010.

[23] J. Brzdęk and J. Sikorska, "A conditional exponential functional equation and its stability," Nonlinear Analysis: Theory, Methods \& Applications, vol. 72, no. 6, pp. 2923-2934, 2010.

[24] J. Sikorska, "On two conditional Pexider functional equations and their stabilities," Nonlinear Analysis: Theory, Methods E Applications, vol. 70, no. 7, pp. 2673-2684, 2009.

[25] J. Sikorska, "On a Pexiderized conditional exponential functional equation," Acta Mathematica Hungarica, vol. 125, no. 3, pp. 287-299, 2009.

[26] J. Sikorska, "Exponential functional equation on spheres," Applied Mathematics Letters, vol. 23, no. 2, pp. 156-160, 2010. 